\title{
The schemes analysis of profile milling of long and non-technological workpieces
}

\author{
Vladimir G. Gusev ${ }^{1}$, Anatoly A. Fomin ${ }^{1, *}$, and Vladimir A. Saldaev ${ }^{2}$ \\ ${ }^{1}$ Institute of Engineering and Automobile Transport, Vladimir State University, Gorky Street 87, \\ Vladimir, 600000, Russian Federation \\ ${ }^{2}$ The Scientific Center for Advanced Energy Technologies, Kazan State Power Engineering \\ University, Krasnoselskaya street 51, Kazan, 420066, Russian Federation
}

\begin{abstract}
The article considers alternative schemes of profile milling of long non-technological workpieces- of the peripheral segments, formed during longitudinal opening of logs. Peripheral segments are a large waste of manufacture and contain the wood fibres with high physics-mechanical and operational properties. Therefore manufacturing qualitative wood products from peripheral segments is especially actual problem. However, the named workpieces are characterised by low adaptability to manufacture, therefore working out and the analysis of the machining schemes, expelling a resonance in technological system are necessary. The non-resonance machining scheme is used in a design of the milling machine tool PFP-100 for profile milling of a large wood waste.
\end{abstract}

Great demand in the world market is used for wood mouldings (block house, lining, euro lining, platbands, etc.) [1], the cross section of which is convex and concave curvilinear contours. The milling of the surfaces of these products with profile cutters is beyond competition with other technological methods due to high productivity and low production costs [2]. A special scientific and economic interest is the technology of manufacturing molded products from large sawmilling waste-peripheral segments S1, S2 (Fig. 1, a), formed as a result of longitudinal cutting of logs. Peripheral segments have a length (3-6) m and are characterized by extremely low workability: an unpredictable shape of the outer surface, a significant and stochastic spread of dimensions and hardness, runout, knotty and lack of developed technological bases (Fig. 1, b) [3], which causes serious difficulties for their machining and leads to extreme cutting conditions [4-6].

${ }^{*}$ Corresponding author: fomin1@mail.ru 


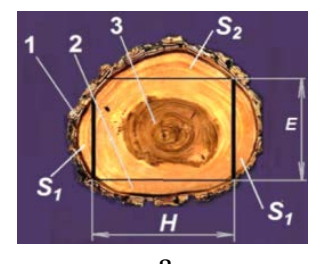

a

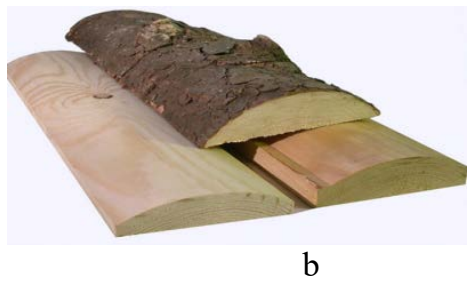

b

Fig. 1. Cross-section of the log (a), untreated segment and processed product (b): 1 - bark, 2 - quality $\log$ fibers, 3 - core, S1, S2 - peripheral segments; H, E are the sizes of a bar.

Sophisticated complex machine systems [7] that provide a comprehensive cutting of logs (at the same time peripheral segments into small elements) are used abroad. After such a cutting of the peripheral segments, its small elements are glued, dried and machined, which leads to a deterioration of the ecological situation (due to the use of adhesives), an extension of the technological chain and a significant increase in the cost of finished products. Prospective is not the use of expensive machine tools, but the development of much cheaper equipment and environmentally friendly processes of profile processing of large sawmill wastes, on the basis of which the release of quality profile products becomes real. The solution of these issues is possible on the basis of deep comprehensive research, development and design work, and, first of all, the development of a vibration-resistant technological profile milling scheme.

The purpose of this study is to develop a profile milling scheme for non-technological large sawmilling wastes characterized by relatively small elastic movements of the workpiece under the action of the workload, a slight vibration speed of the elastic recovery of the wood, and less severe impacts of the cutting teeth of the cutter on the workpiece surface. To achieve the goal, it is necessary to solve the following tasks: development of alternative profile milling schemes for peripheral segments; analysis and choice of the scheme for practical implementation; experimental verification of the selected scheme; development of recommendations on the further use of research results.

In the logging process, logs are cut down, branches are cut, transported along the ground, as a result of which the outer surface of the log contains nonmetallic inclusions (sand, clay, etc.), which adversely affects the resistance of the cutting tool during profile milling. The oncoming milling scheme, unlike the passing one, is characterized by less dynamic impact of the mill on the workpiece, and its teeth are not encountered when processing foreign abrasive inclusions, which provides favorable cutting conditions. For these reasons, the material presented hereafter refers to the on-coming profile milling of segments. Due to the large extent of the machined surfaces of the peripheral segments, the use of a tool for rigid fixing of the workpiece is not practical due to the considerable complexity of the technological equipment. Therefore, alternative profile milling schemes were developed, in which:

- the peripheral segment (workpiece) is supported by several rollers, between which there is no intermediate support (Fig. 2) (first diagram);

- in the middle between the rollers a plate 4 is fixed, forming a gap Sp with the technological base of the workpiece (Fig. 3) (second scheme);

- a pulley 8 is installed between adjacent rollers, which is in contact with the workpiece setting base of the workpiece (Fig. 4) (the third scheme).

In the process of profile milling of the unpeeled curvilinear surface of the peripheral segment 1 (Fig. 2), an allowance of thickness $t$ is removed. 


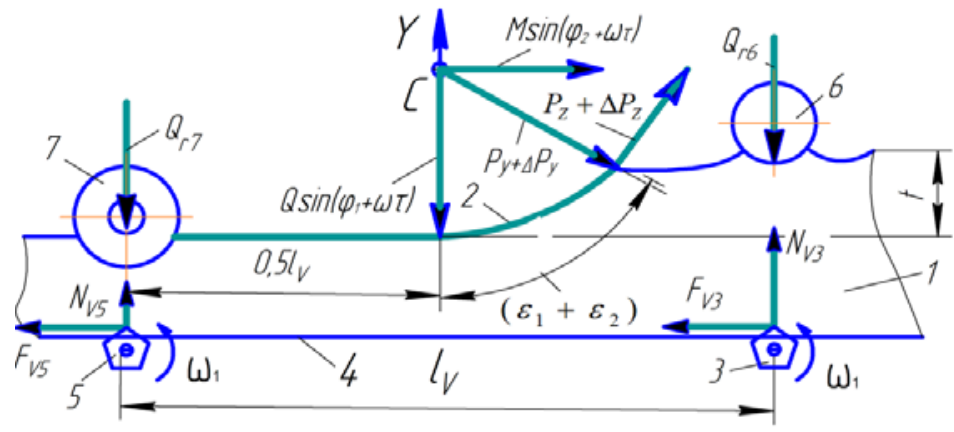

Fig. 2. Scheme of treatment without intermediate support of the workpiece.

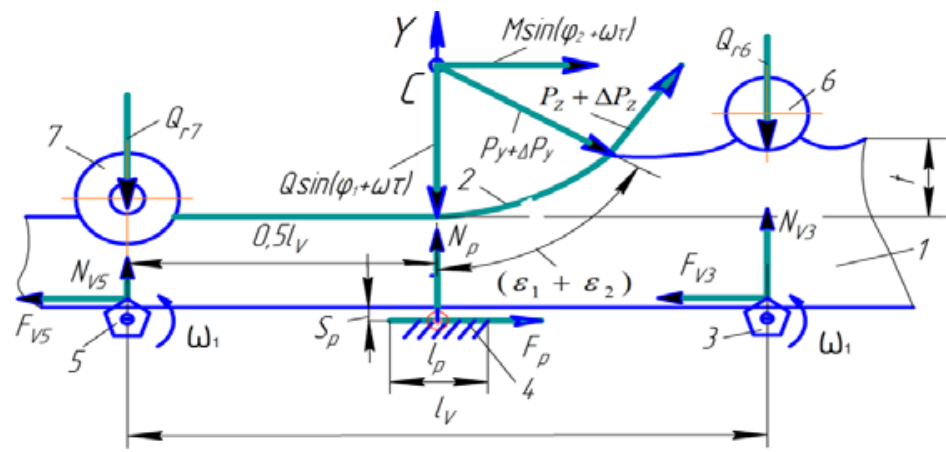

Fig. 3. Scheme of treatment with an intermediate support between the rolls, made in the form of a plate.

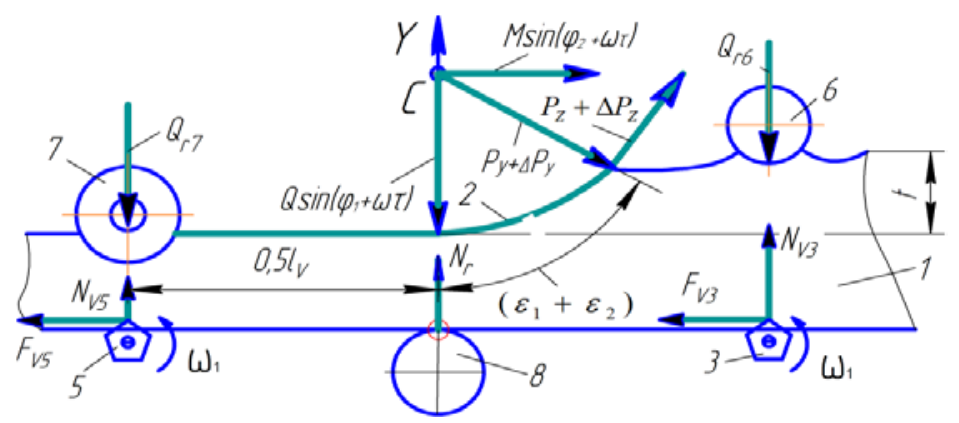

Fig. 4. Scheme of treatment with the installation of the support pulley between the rollers.

The main $\left(P_{Z}+\Delta P_{Z}\right)$ and radial $\left(P_{y}+\Delta P_{y}\right)$ components of the cutting force act on the processed segment, which are characterized by the increments $\Delta P_{Z}$ and $\Delta P_{y}$ due to the variability of the allowance, hardness and knotty. After a single cutting with the tooth of the cutter, the workpiece has a trace in the form of a curve of line 2 . The centrifugal force $Q \sin \left(\omega \tau+\varphi_{1}\right)$ also acts on the workpiece ( $\omega$ is the angular velocity of the mill, $\tau$ is the current machining time, $\varphi_{1}$ is the initial angular position of the force $Q$ ), caused by the principal vector of imbalances $D s t$, and the bending moment $M \sin \left(\omega \tau+\varphi_{2}\right)\left(\varphi_{2}\right.$ is the angular reference of the moment $M$ ), due to the main moment of the instrument $M_{D}$ imbalances. The force $Q$ and the moment $M$ change during processing by sinusoidal law.

The maximum load on the spindle unit with the tool occurs when the main vector Dst is perpendicular to the $M_{D}$ main imbalance moment. The peripheral segment 1 , mounted on the technological base surface 4 , moves with the speed of the working feed when the bending 
rolls 3 and 5 rotate with angular velocity $\omega_{1}$. When the segment 1 is pressed by rollers 6 and 7 with the forces $Q_{r 6}$ and $Q_{r 7}$, the teeth of the rolls are cut into the surface 4 and the drive forces $F_{V 3}$ and $F_{V 5}$ are transmitted to the segment. The roller 6 is pointed, therefore, under the action of the force $Q_{r 6}$, it cuts into the workpiece, ensuring its direction when entering the processing zone. There is a normal reaction $N_{V 3}$ of the roll 3 .

The profiled roller 7 directs the segment at the exit from the treatment zone without damaging its treated surface. Changes in the elastic displacements of the segment under the influence of a variable workload lead to a change in the frictional forces of the segment with the bending rolls 3,5 and the rollers 6,7 .

In the absence of support between adjacent rollers (the first scheme), there is a danger of resonance due to the coincidence of the natural frequency of the transverse bending vibrations of the workpiece with the frequency of external dynamic action, therefore, before processing it is necessary to perform calculations for the absence of resonance.

The frequency of natural oscillations of the workpiece as a function of its dimensions, modulus of elasticity and other parameters is determined by the formula:

$$
f_{c}=\frac{\left(h_{n}+h_{o}\right)}{l_{v}} \sqrt{\frac{E\left(h_{n}+h_{o}\right)\left(B_{n}+B_{o}\right)}{l_{v} M_{k}}}
$$

where $h_{n}, h_{o}, B_{n}, B_{o}$, respectively, is the height and width of the untreated and processed part of the peripheral segment; $E$ - modulus of elasticity of wood; $l_{v}$ - interaxial distance of adjacent rollers; $M_{k}$ is the mass of the workpiece that participates in the oscillatory process.

From (1) it follows that the value of the natural oscillation frequency $f_{c}$ of the workpiece can be controlled, and, consequently, also the vibration resistance of the profile milling process of the peripheral segment. The frequency of external dynamic impact during profile milling is determined by the formula:

$$
f_{v}=n z / 60
$$

where $n$ is the rotational speed of the mill; $z$ - number of teeth of the cutter.

Absence of resonance in the technological system of profile milling of workpieces is achieved by meeting the conditions:

$$
f_{v} \leq(0,6-0,7) f_{\mathrm{c}}
$$

From the expressions (1)-(3) we determine the working frequency of the shaping cutter rotation, at which there is no resonance in the technological system:

$$
n \leq(2,16-2,52) \cdot 10^{3} \cdot \frac{\left(h_{n}+h_{o}\right)}{z l_{v}} \cdot \sqrt{\frac{E\left(h_{n}+h_{o}\right)\left(B_{n}+B_{o}\right)}{l_{v} M_{k}}} .
$$

With the initial information about the geometric dimensions of the peripheral segments, the elasticity modulus of the wood, the oscillating mass, the interaxial distance of the adjacent rolls, it is possible to determine by (4) the resonanceless operating speed of the cutting tool in $\mathrm{min}^{-1}$ for specific profile milling conditions.

Calculations according to (2) show that, when processing peripheral segments with a cutting speed $(45 \ldots 50) \mathrm{m} / \mathrm{s}$, the number of cutting teeth of the milling cutter $z=4 \ldots 10$ the frequency of the compulsive force is in the interval $(330 \ldots 830) \mathrm{Hz}$. Resonance can be avoided with a small interaxial distance of the adjacent rollers $l_{v}=(0.2 \ldots 0.5)$ meter and processing workpieces of relatively large sizes $h_{n}, h_{o}, B_{n}, B_{o}$ (100 mm or more), but setting the rolls at a small distance $l_{v}$ complicates the mechanism of the workpiece feed, and the increase in its size narrows the technological possibilities of the first scheme. 
On the basis of the analysis, the drawbacks of the first scheme are revealed: a change in the friction forces within a wide range, which increases the probability of occurrence of selfoscillations in the technological system; The contact of the tool with the workpiece takes place under harsh conditions, when the milling cutter and the workpiece move towards each other in the process of resilient restoration; nonresonant processing is possible with a small interaxial distance $l_{v}$ of the rolls and a relatively large size of the workpiece, which limits the technological possibilities of the circuit. Let us analyze the second scheme (Fig. 3). The length of the support plate 4 is $l_{p}$, the rolls 3,5 support the workpiece 1 , creating normal reactions $N_{V 3}, N_{V 5}$. The plate 4 limits the elastic movements of the workpiece with the value of the static gap $S_{p}=$ [y] between the plate and the process base of the segment. The pressing of the profile roller 7 to the processed surface of the segment provides a spatial position of the longitudinal axis of the segment and eliminates its displacement when shear forces occur, for example, in the processing of knots.

In the absence of the cutting process, the normal reactions $N_{V 3}, N_{V 5}$ are determined by the degree of compression of the springs and their rigidity $d_{r 6}$ and $d_{r 7}: N_{V 3}=Q_{r 7} ; N_{V 5}=$ $Q_{r 6}$. The approximate total value of the frictional force between the rolls, rollers and workpiece in the absence of the cutting process equals to:

$$
\mathrm{F}_{t r}=f_{1}\left(Q_{r 6 .}+Q_{r 7}+N_{V 3}+N_{V 5}\right),
$$

where $f_{l}$ - coefficient of rolling friction of wood against metal.

The frictional force between the plate 4 and the installation plane of the segment 1 is:

$$
F_{p}=f_{2} N_{p}
$$

where $f_{2}$ - coefficient of sliding friction of wood against metal; $N_{p}$ - normal reaction of the plate. Since the support plate takes part of the external load, the frictional forces and the external load on the rolls 3, 5 are smaller in the second scheme than in the first one. The total frictional force consists of friction forces of the workpiece with rolls, plate and rollers:

$$
\mathrm{F}_{t r 2}=\mathrm{F}_{p}+\mathrm{F}_{V 3}+\mathrm{F}_{V 5}+\mathrm{F}_{r 6}+\mathrm{F}_{r 7} .
$$

The friction force $F_{t r 2}$ is greater than the friction force that occurs during the first processing scheme. On the level of elastic movement of the workpiece it is preferably better using the second scheme than the first one, since the elastic restoring forces of the workpiece are smaller in the second case and equal to:

$$
y_{1} d_{c}>S_{p} d_{c}
$$

where $y_{1}$ - elastic movements of the workpiece during the first scheme of segment processing, $d_{c}$ - the stiffness of the segment in the vertical direction.

Smaller elastic forces affect the decrease in vibration speed of the elastic restoration of wood, which leads to a less severe impact of the workpiece when it meets the cutting teeth of the mill. The components $F_{V 3}, F_{V 5}, F_{r 6}, F_{r 7}$ can be reduced by setting the rolls 3,5 and the rollers 6,7 on the rolling bearings. The second scheme, in comparison with the first one, provides a more relaxed profile milling of peripheral segments, which is its advantage.

Reduction of the numerical value of the frictional force is possible by using, instead of the plate 4, a roller 8 (figure 4) mounted on the rolling bearing. As a result, we get the third processing scheme. The normal reaction $N_{V 3}$ of the roll 3 in the absence of cutting is equal to the force $Q_{r 6}$. The profiled roller 7 presses on the machined surface with a force $Q_{r 7}$, which facilitates the infeed of the roll 5 into the mounting surface of the peripheral segment. Since the workpiece has a support in the form of a roller mounted on the rolling bearing, the frictional force in the support is insignificant. The impact pulse of the cutter tooth is perceived 
by a low frictional force, which leads to a selection of gaps in the kinematic elements of the feed chain and to the elastic twisting of the shafts under the action of the cutting torque.

After the cutting tooth emerges from contact with the segment, the cutting torque is zero. The process of elastic restoration of the deformed elements of the kinematic supply chain and cutting tool begins, and the elastic forces are added with the feed force $\left(F_{V 3}+F_{V 5}\right)$, which causes an increase in the speed of the translational motion of the workpiece towards the shaped cutter. The interaction of the next cutter tooth with wood occurs in unfavorable conditions, when the speed of the workpiece movement with respect to the tool is maximal. A situation is created that is similar to the first processing scheme, with the only difference being that in the first case resonance oscillations occur in the vertical plane, and in the third scheme - in the horizontal plane. The milling process under the third scheme becomes unstable [8].

To verify this situation, experiments were carried out. During the profile milling of the peripheral segment, according to the third scheme, resonant phenomena were observed when the workpiece, making intensive oscillations in the horizontal plane, came out of contact with the shaping cutterl in the direction opposite to the direction of the working feed, after which it moved with increasing speed to the cutting tool. There was a blow to the teeth of the cutter on the workpiece, as a result of which the workpiece again came out of contact with the cutter.

After inspecting the contact points of the installation reference plane of the workpiece with the drive rolls, it was found that the traces of the cutting teeth of the drive rolls were not inserted into the wood, that is, the "teeth" of the wood formed by pressing the metal rolls into the workpiece were cut off. The described picture was due to insufficient resistance to the rapid elastic recovery of the kinematic supply chain elements. The process was characterized by intensive impact of the tool on the workpiece, there was a real danger of breakage of the cutting teeth of the mill, so this treatment processing was stopped.

Thus, the non-resonant profile milling of the peripheral segments is provided to the greatest extent by the second scheme, in which the additional support of the workpiece is made in the form of a metal plate installed in the cutting zone opposite to the shaped cutter.

Mathematical dependencies (1) - (8) were used in the development of a woodworking machine with software control of the PFP-100 model, in which the second scheme of profile milling of peripheral segments and stemwood was implemented. Technical and technological solutions, used in the machine tool, are protected by patents of the Russian Federation [9, 10]. The real machine model was certified, presented at the 6th Moscow International Salon of Innovations and Investments, where it was awarded a diploma and a silver medal.

\section{References}

1. A.A. Fomin, V.G. Gusev, Multidimensional Model of Surface Waviness Treated by Shaping Cutter, Procedia Engineering, 206, pp. 286-292 (2017)

2. A.A. Fomin, The equipment and production engineering of machining of a wood waste (Engineering industry, Moscow, 2013)

3. A.A. Fomin, Determining undeformed chip thickness models in milling and its verification during wood processing, Solid State Phenomena, 265, pp. 598-605 (2017)

4. A.A. Fomin, Kinematics of surface formation in milling, Russian Engineering Research, 33(11), pp. 660-662 (2013)

5. V.G. Gusev, A.A. Fomin and A.R. Sadrtdinov, Dynamics of stock removal in profile milling process by shaped tool, Procedia Engineering, 206, pp. 279-285 (2017) 
6. Xiang Su, et al, Predictive model of milling force for complex profile milling, The International Journal of Advanced Manufacturing Technology, 87(5-8), pp. 1653-1662 (2016)

7. Y. Zhou, Z.C. Chen, J. Tang \& S. Liu, An innovative approach to NC programming for accurate five-axis flank milling of spiral bevel or hypoid gears, Computer-Aided Design, 84, pp. 5-24 (2016)

8. Y. Stepanov, V. Gordon, S. Ovsiannikova, Influence of gyroscopic effect on the critical speed of the rotation of the heterogeneous rod, ICSV, v.2, pp. 892-897 (2006)

9. A.A. Fomin, V.G. Gusev, The machine tool for slab preprocessing, Patent 95589, (2010)

10. A.A. Fomin, V.G. Gusev, A way of a roughing-out of a slab, Patent 2443547, (2012). 